\title{
Applications and Performance of a Nanoreceiver with a Carbon Nanotube Antenna Forest
}

\author{
C. Emre Koksal, Eylem Ekici
}

\begin{abstract}
We introduce an RF nanoreceiver, based on a forest of carbon nanotube (CNT) antennas packaged together. Based on the physical model of a charged carbon nanotube under RF exposure, which is significantly different from a typical RF antenna, we analyze the performance of our nanoreceiver. We show that, for low signal-bandwidth applications, our nanoreceiver is highly robust, allowing for a continual operation over extended periods of time at low probabilities of error at reasonably low SNR values. For the high signal-bandwidth applications, we show that, at a given SNR, the achieved rate grows as $n^{1 / 4}$ with the number, $n$, of the CNTs, at reasonably low probabilities of error. Due to extremely small scale of the CNTs, many millions of CNTs can be packed into very small areas (e.g., hundreds of millions in $1 \mathbf{m m}^{2}$ ) to achieve rates comparable to the commercially available wireless receivers. Hence, a significant spatial miniaturization is achieved by our nanoreceiver compared to a classical RF receiver without a loss in the achievable rates.
\end{abstract}

Index Terms - nanocommunication, nano-scale networks, carbon nanotube transceivers, detection theory

\section{INTRODUCTION}

Nanoscale systems are envisioned for many crucial future applications ranging from targeted medicine and drug delivery to high fidelity sensors [1]-[5]. Miniaturization of communications systems has been in the forefront of research and development efforts for decades. Until very recently, communication system sizes and design alternatives were considered limited by the circuit, battery, but most importantly, antenna sizes. For instance, the use of common operating frequencies of $100 \mathrm{~s}$ of $\mathrm{MHz}$ to several $\mathrm{GHz}$ meant an antenna size in the order of centimeters. Such limitations render incorporation of communication into nanoscale systems impossible. Electromagnetic communication in such scales is reserved for the $\mathrm{THz}$ range, which has extremely high attenuation through matter [6]. Therefore, traditional EM-based communication had very limited use in nanoscale devices and systems. Instead, other paradigms were considered to facilitate communication of nanoscale devices for short distances [1], [7] via molecular communication and longer distances [8], [9] via bacteria and nano-motors.

Recently, new EM-based radio receivers and transmitters have been proposed using Carbon Nanotubes (CNTs) [10], [11]. These communication systems are fundamentally different from traditional antenna-based systems: Rather than relying on the oscillation of electrons inside the antenna in response to EM waves, CNTs oscillate themselves when they are charged. Oscillations lead to variation of the distance

C. Emre Koksal and Eylem Ekici are with the Department of Electrical and Computer Engineering, The Ohio State University, Columbus, OH (E-mail: $\{$ koksal, ekici\}@ece.osu.edu). of the tip of the CNT from a cathode plate. The distance variations are then detected as fluctuations of the emission current. CNT-based receiver systems have been validated in implementation [10] and further analyzed in [12]. The groundbreaking property of CNT-based communication systems is that it is possible to establish communication in the 100 s of $\mathrm{MHz}$ range with systems that are 100s of nm in size. Even more recently, we have developed a communication-theoretical analysis of CNT-based receiver systems [13], [14]. Almost concurrently, system and networking aspects of nanoscale devices with CNT-based radios have been discussed in [15].

In this work, we analyze the behavior of forests of CNT antennas, which are composed of thousands or more CNTs vertically aligned on the same substrate. Using the unique response characteristics of charged CNTs under RF exposure, we introduce two threads of analysis, highlighting two distinct use cases of CNT-based nano communication systems. For the low signal-bandwidth applications, we show that our proposed receiver system is highly robust, allowing for a continual operation over extended periods of time at low probabilities of error at reasonably low SNR values. For the high signalbandwidth applications, we show that the achieved rate grows as $n^{1 / 4}$ with the number, $n$, of the CNTs, at reasonably low probabilities of error at a given SNR. Since CNT antennas can be packed very densely (e.g., hundreds of millions in 1 $\mathrm{mm}^{2}$ ), a significant spatial miniaturization is achieved by our nanoreceiver compared to a classical RF receiver without a loss in the achievable rates.

\section{PHYSICAL MODEL}

The basic operation of the CNT-based nanoreceiver is illustrated in Fig. 1. It consists of a CNT attached to an anode with the other end free to move. CNTs behave like cantilevers with high elastic strength, and an elastic constant in $100 \mathrm{GPa}$ range. A longitudinal electric field applied on a CNT induces a charge density at the tip of the CNT. Under electromagnetic excitation, the oscillating electric field perpendicular to the CNT exerts an oscillating force on it and causes it to move. Near the resonance frequency $f_{0}$ of the CNT cantilever, EM energy couples most efficiently into the CNT, and the CNT vibrates with a large amplitude. Fundamental properties such as mass, length, and diameter determine the resonance frequency and quality factor $(Q)$ of the cantilever vibration. Overall, the resonance frequency is inversely proportional to the square of the length and the square root of the cross section area of the CNT. The response of the vibration amplitude to an oscillating electric field perpendicular to the CNT is proportional to 


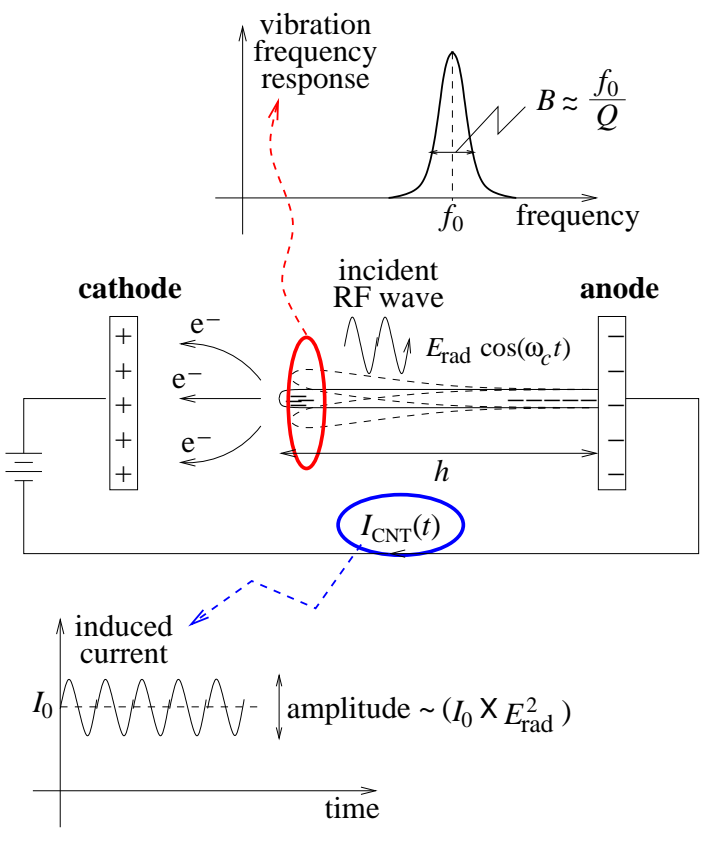

Fig. 1. Basic operation of a CNT receiver

$\frac{E_{\text {rad }}}{\sqrt{\left(f_{c}^{2}-f_{0}^{2}\right)^{2}+\left(f_{c} f_{0} / Q\right)^{2}}}$, where $f_{0}$ is the resonance frequency of the CNT, $f_{c}$ and $E_{\text {rad }}$ are the carrier frequency of the signal acting on the CNT and its amplitude, respectively, and $Q$ is the quality factor. The response of the vibration amplitude peaks at $f_{c}=f_{0}$ and dies off rather fast as $f_{c}$ moves "out of tune" from the resonance frequency $f_{0}$, as shown in Figure 1. A simple theoretical model for electromagnetic excitation of CNTs is provided in [10].

The electric field between the free tip of the CNT and the cathode causes electrons to jump across the gap and results in tunneling current. The tunneling current varies exponentially with the distance between the tip and the cathode, governed by the Fowler Nordheim equation. Since the tunneling current depends exponentially on the inverse of the distance, small deviation of the CNT due to bending can lead to large changes in the tunneling current. The tunneling current resulting from the oscillation of the CNT has also been depicted in Figure 1. Here, the variation in the current is proportional to the square of $E_{\text {rad }}$ [10], [13] and is amplified by a factor, $I_{0}$, identical to the tunneling current observed when the CNT is at rest. This leads to a square-law behavior for the observed signal with respect to the incident radiant electric field strength.

\section{NANORECEIVER MODEL}

The block diagram of our nanoreceiver is illustrated in Fig. 2. The nanoreceiver is composed of two parts: the front end and the energy detector. The basic components of the front end summarizes the physics of the charged CNTs under and incident RF wave. The front end includes $n$ parallel CNT antennas and $n$ associated square-law devices followed by an amplifier each. Here, $h_{\mathrm{CNT}, j}(t)$ is the impulse response of the linear filter that captures the input-output behavior of the $j$ th CNT antenna, where the input $s(t)$ is the electric field intensity of the incoming RF wave and the output $Y_{o, j}(t)$ is the amplitude of the associated vibrations in CNT $j$. The magnitude response of the $j$ th CNT antenna is:

$$
H_{\mathrm{CNT}, j}(f)=\left|\frac{Y_{o, j}(f)}{S(f)}\right|=\frac{q / m_{\mathrm{eff}}}{4 \pi^{2} \sqrt{\left(f^{2}-f_{c, j}^{2}\right)^{2}+\left(f f_{c, j} / Q\right)^{2}}},
$$

where $f_{c, j}$ is the resonance frequency of the $j$ th CNT antenna, $q$ is the charge at the tip, and $m_{\mathrm{eff}}$ is the effective mass of the CNT. We assume the length of each CNT antenna to be random. We model this randomness using a normal distribution, i.e., $f_{c, j} \sim \mathcal{N}\left(f_{0}, \sigma_{f_{0}}^{2}\right)$, independently of $f_{c, j^{\prime}}$ for all $j^{\prime} \neq j$. The 3 -dB bandwidth, $B$, of the antenna response $H_{\mathrm{CNT}, j}(f)$ can be found by solving $H_{\mathrm{CNT}, j}\left(f_{c, j}\right) / \sqrt{2}=$ $H_{\mathrm{CNT}, j}\left(f_{c, j}+B / 2\right)$. With the additional assumption that the the bandwidth is much smaller than the center frequency, $B \ll f_{0}$ (which is highly reasonable since the CNT have a narrow pass-band), one can find $B \approx \frac{f_{0}}{Q}$ for all the CNTs.

The assume that the signal is corrupted by noise at two levels: The acoustic noise, $W_{a, j}(t)$, is the mechanical component caused by radio static within the band of the antenna. Acoustic noise directly affects the amplitude of the vibrations $Y_{o, j}(t)$. The second one is the thermal noise, $W_{T}(t)$, due to the electronic components of the detector and it is additive on the total detected current. We assume both the acoustic and the thermal noise are additive white Gaussian. In our analysis, we assumed that the acoustic noise is the dominant noise source for two reasons: 1$)$ the size of the CNT forest is large $(n \gg 1)$ in our systems; 2) every branch of the front end contains an amplifier with a very large gain (e.g., this gain is reported to be at the range of $50 \mathrm{~dB}$ in [10]).

The second component of the nanoreceiver is the energy detector. Since the signal $\sum_{j=1}^{n} I_{r, j}(t)$ is the current at the output of the front-end of the receiver, the integrator can be realized by a mere capacitor. The integrator is followed by the sampler, sampling the output of the integrator once every $T$ seconds (symbol period), i.e., the data rate is $1 / T$ bits/sec. Each sample $Y_{s}[k]$ is compared with a pair of predetermined thresholds $\tau_{1}$ and $\tau_{2}$ and a ' 0 ' or a ' 1 ' bit is decoded depending on these comparisons.

For information transmission, we assume that the simple ON-OFF Keying (OOK) signalling scheme is used. Apart from the simplicity of the scheme, there are other reasons that make OOK suitable for our nanoreceiver design. In OOK, during the ON period, the transmitter transmits pure sinusoids of duration $T$, i.e., for each antenna $j$, we have $s(t)=a \cos \left(2 \pi f_{0} t+\phi\right)$, where $\phi$ is the random phase. Depending on the rate of information transmission desired by the application, the bandwidth of the input signal $s(t)$ can be large or small compared to the CNT bandwidth. The performance of our nanoreceiver under the low signalbandwidth regime was studied in [14]. Here, we illustrate the performance under the high signal-bandwidth regime as well, and discuss the fundamental differences between the two.

The square-law device at each branch acts as a demodulator for the signal component (filtered by the antenna response $\left.h_{\mathrm{CNT}, j}(t)\right)$ of each waveform $Y_{o, j}(t)$. This avoids the need for complicated components such as the phase locked loop to be implemented at the nano scale. The energy detector integrates $\sum_{j=1}^{n}\left(I_{\mathrm{CNT}, j}(t)\right)+W_{T}(t)$ over $T$ and a sampler 


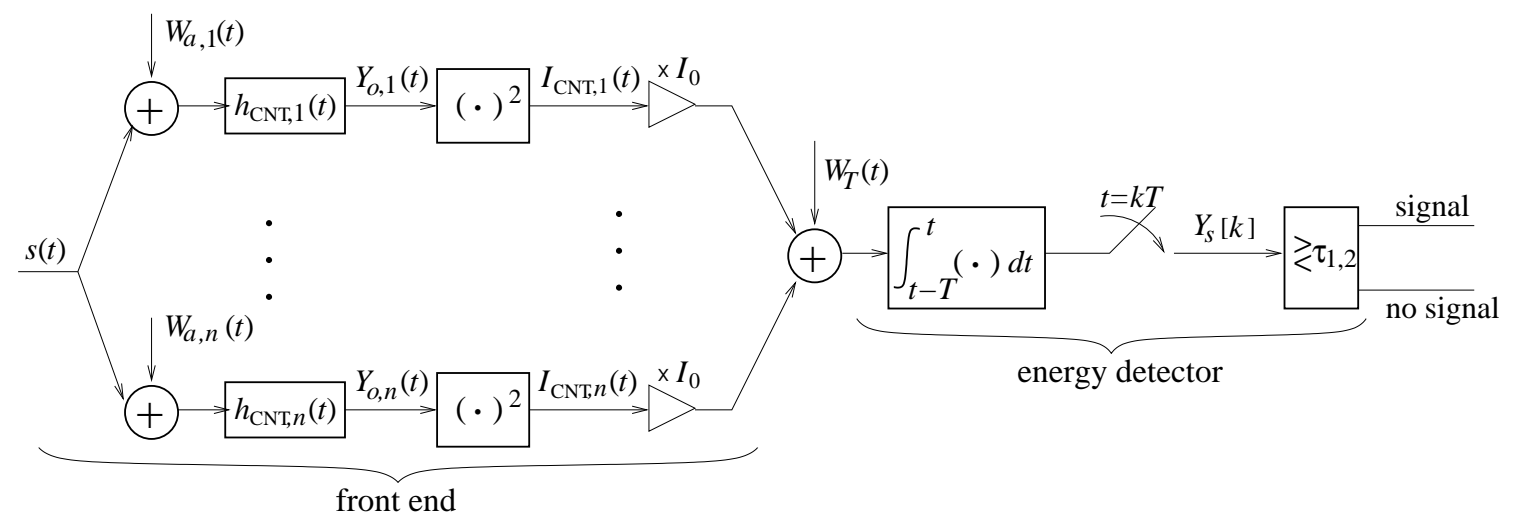

Fig. 2. System model of our nanoreceiver.

samples the output of the integrator every $T$ seconds. Due to the square-law device, there are three components of $I_{\mathrm{CNT}, j}(t)$ under the activation attempt: signal component; signal-noise cross component, which is a Gaussian process; and noise-noise cross component, which has Chi-squared samples. Under both low signal-bandwidth and the high signal-bandwidth regimes, we assume that the thresholds of the detector are chosen optimally, to minimize the probability of error. Hence, we use a maximum a posteriori or maximum likelihood decision rules.

\section{Applications and Performance}

We consider two different types of applications, depending on the bandwidth of the RF signal. In low signal-bandwidth regime, the signal bandwidth is much smaller than the bandwidth of the CNTs. Given that the typical CNT bandwidth varies between tens to hundreds of $\mathrm{kHz}$ [13], the low bandwidth regime allows for applications at rates lower than a few kbps. The candidate applications include the activation of nanosystems, in which the system needs to remain inactive for a period of time and is expected to become active only when it receives an activation signal. The main performance goal in such applications is to simultaneously achieve low probabilities of false activation and unsuccessful activation. Consequently, the nanoreceiver can operate reliably over extended periods of time without observing an undesirable false activation, but becomes active correctly upon receiving an activation signal.

In high signal-bandwidth regime, the signal bandwidth is much larger than the bandwidth of the CNTs. In this regime, our objective is to achieve data rates, comparable to those of traditional RF receivers. We first analyze the achievable data rates as a function of the number of CNT antennas. Note that, due to extremely small scale of the CNTs, many millions of CNTs can be packed into very small areas (e.g., hundreds of millions in $1 \mathrm{~mm}^{2}$ ). Exploiting this large number of CNT antennas, we show that it is possible to achieve data rates comparable to the commercially available wireless receivers at reasonably low bit error rates. Hence, a significant spatial miniaturization is achieved by our nanoreceiver compared to a classical RF receiver without a loss in the achievable rates.

1) Low Signal-Bandwidth Regime: In this regime, we consider applications which do not require a high data rate, but a robust operation for extended amounts of time. In particular, in certain applications, nanosystems can be activated by

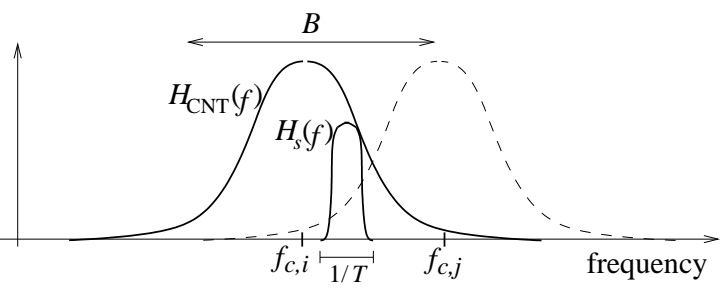

low signal-bandwidth

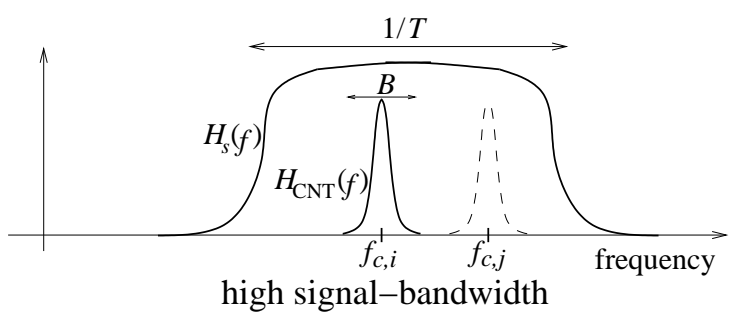

Fig. 3. Spectra of the signal and the CNT for high signal-bandwidth and low signal-bandwidth regimes

activation signals sent remotely so that they initiate various tasks. For instance, nano-scale agents that are injected in human body can be used for targeted drug delivery. Once the activation signal is detected, they can release the drug. In such applications, the critical issue is the reliable operation for extended durations, rather than a high-data rate. Thus, in this regime, we assume the duration, $T$, of the activation signal to be large, i.e., $T \gg 1 / B$, where $B$ is the bandwidth of a CNT.

The shapes of the spectra, $H_{s}(f)$ and $H_{\mathrm{CNT}}(f)$, of the activation signal and a CNT, respectively, are illustrated in Fig. 3. There, one can see that the variations in the resonance frequency of the CNTs significantly affect the amount of signal energy that the system absorbs. As shown in Fig. 3, while the narrowband signal lies well in the band of CNT $i$, it is outside of the pass-band of CNT $j$, eliminating its contribution to the received energy.

To evaluate the performance of our nanoreceiver in the low signal-bandwidth regime, we analyze the probabilities of the error events. In particular, we evaluate $p_{u a}$ and $p_{f a}$, which denote the probabilities of unsuccessful and false activation respectively. We also evaluate the overall probability of error, $p_{e}=p_{a} p_{u a}+\left(1-p_{a}\right) p_{f a}$, where $p_{a}$ denotes the probability of an activation signal in a given period of duration $T$. To find 


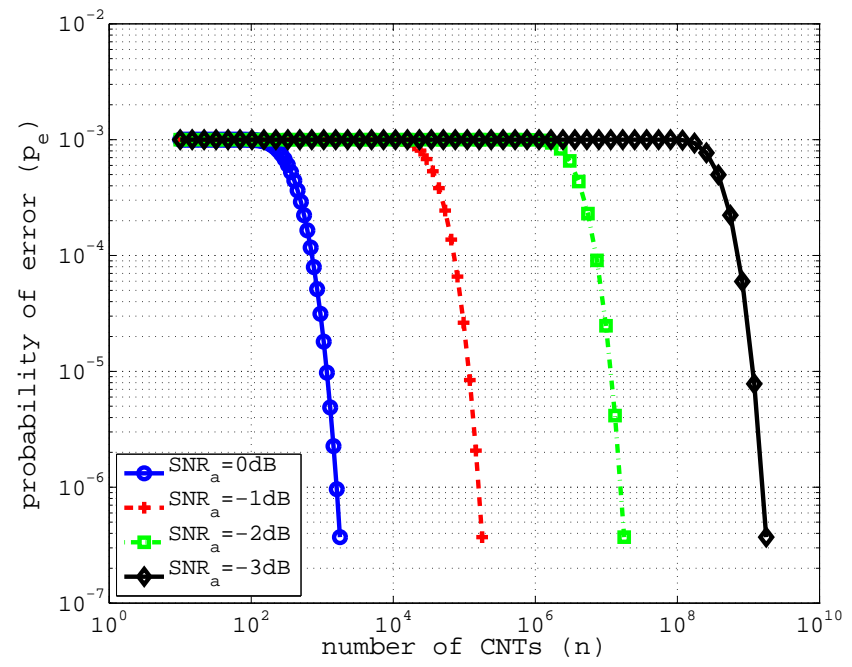

(a) Error probability vs. number of CNTs

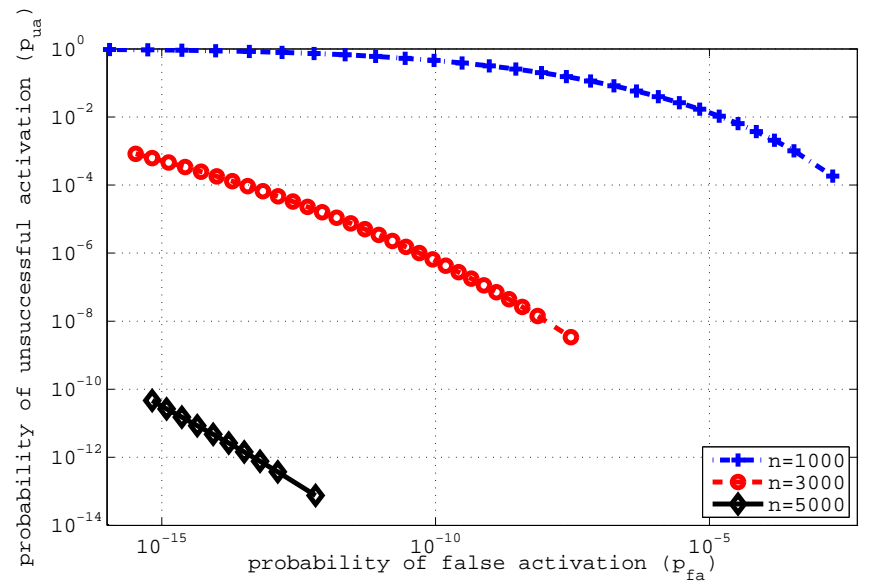

(b) Receiver operating characteristics

Fig. 4. Performance evaluation of the nanoreceiver in the low signalbandwidth regime.

the error probabilities, we use the thresholds of our detector, corresponding to the maximum a posteriori (MAP) detection. We illustrate the associated performance in Fig. 4. There, the parameter values are $f_{0}=15 \mathrm{MHz}, Q=500$, which implies that the average CNT bandwidth $B=30 \mathrm{kHz}$. Also, activation probability is $p_{a}=10^{-3}$, activation period length is $T=$ $0.1 \mathrm{sec}$, and the ratio of the standard deviation of the CNT lengths to the bandwidth is $\frac{\sigma_{f_{0}}}{B}=\frac{1}{3}$.

In Fig. 4(a), we plot the probability of error, $p_{e}$, as a function of the number of antennas for various values of $\mathrm{SNR}_{a}$. It is notable that, even for a low $\mathrm{SNR}_{a}$, it is possible to achieve low probabilities of error with reasonably small number of CNTs. For instance, approximately $n=1000$ CNTs is sufficient to achieve a $p_{e}$ of $10^{-5}$. Another important conclusion is that, the performance is highly sensitive to the variations of $\mathrm{SNR}_{a}$. For instance, even to make up for a $1 \mathrm{~dB}$ drop in $\mathrm{SNR}_{a}$, we need to increase the number of CNTs by a factor of 100 . While a certain $\mathrm{SNR}_{a}$ is sufficient to achieve a low error probability, a slight decrease in $\mathrm{SNR}_{a}$ causes a huge performance drop. This phase-transition-like phenomenon we observe in the error probability can be explained as follows. Due to the large number of CNTs, a substantial averaging of the acoustic noise occurs when the signals induced by the CNTs are combined at the output of the front-end. The cumulative noise, averaged over all the CNTs is almost deterministic. Thus, if the signal power is below that level, probability of error is high (with uncoded OOK modulation), but once the signal power exceeds that critical threshold, the probability of error becomes almost 0 .

In Fig. 4(b), we plot the receiver operating characteristics (ROC), i.e., the tradeoff between probabilities $p_{u a}$ and $p_{f a}$. In this plot, the signal to acoustic noise ratio is $\mathrm{SNR}_{a}=0 \mathrm{~dB}$. From the figure, one can deduce that $n=3000$ CNTs is sufficient for 1 month of continual operation with $p_{u a}=10^{-7}$ at a cumulative probability of false activation $p_{f a}<10^{-2}$ over the entire month. This example shows that the nanoreceiver can be highly reliable in the low signal-bandwidth regime, even with a few thousand CNTs.

2) High Signal-Bandwidth Regime: In this regime, our ultimate purpose is to use our nanoreceiver to achieve a data rate, comparable to those achievable by traditional RF receivers. Since the bandwidth of a typical CNT is no more than a few hundreds of $\mathrm{kHz}$, in this regime, the duration, $T$, of a symbol is much smaller than the CNT bandwidth, i.e., $T \ll 1 / B$.

The shape of the spectra, $H_{s}(f)$ and $H_{\mathrm{CNT}}(f)$, of a symbol and a CNT, respectively, are illustrated in Fig. 3. Each CNT has a relatively narrow bandwidth and, unlike the low bandwidth regime, it will only absorb a small portion of the signal energy. In this regime, the signal spectrum is approximately constant along the range of resonance frequencies of CNTs. Thus, one can see that the variations in the resonance frequency of the CNTs do not significantly affect the amount of signal energy each CNT absorbs.

To evaluate the performance of our nanoreceiver in the high signal-bandwidth regime, we analyzed the bit error rate (BER). Here, the data rate is $1 / T$ and to evaluate the BER, we select the thresholds of our detector, corresponding to the maximum likelihood (ML) detection. We illustrate the associated performance in Fig. 5. There, the parameter values are $f_{0}=15 \mathrm{MHz}, Q=500$, which imply that the average CNT bandwidth is $B=30 \mathrm{kHz}$. The ratio of the standard deviation of the CNT lengths to the bandwidth is $\frac{\sigma_{f_{0}}}{B}=\frac{1}{3}$, but since the signal bandwidth, $1 / T \gg B$, the variation of the CNT resonance frequencies does not have any major impact on the performance.

In Fig. 5(a), we plot the BER as a function of the number of CNTs at a data rate of $1 \mathrm{Mbps}$ (i.e., $T=10^{-6}$ ). Here, the number of CNTs necessary to achieve a similar performance to the low signal-bandwidth regime is significantly larger. This is plausible, since, unlike the low signal-bandwidth regime, in this regime, each CNT can only absorb a small portion of the signal power. However, the result is still optimistic, since the number of CNTs to achieve a reasonable performance is feasible in the current state of the nano-manufacturing technology. For instance, the size of the CNT forest necessary to achieve a BER of less than $10^{-6}$ at $\mathrm{SNR}_{a}=-3 \mathrm{~dB}$ is $n=10^{6}$, which can be planted in an area as small as $0.1 \mathrm{~mm}^{2}$.

Also, if we compare Fig. 5(a) with Fig. 4(a), we can see that, 


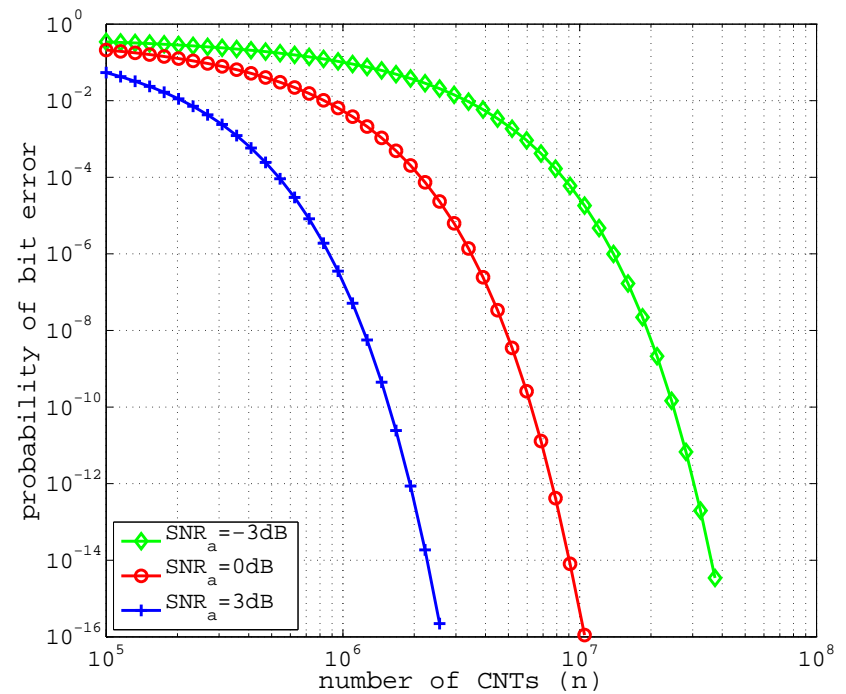

(a) Error probability vs. number of CNTs

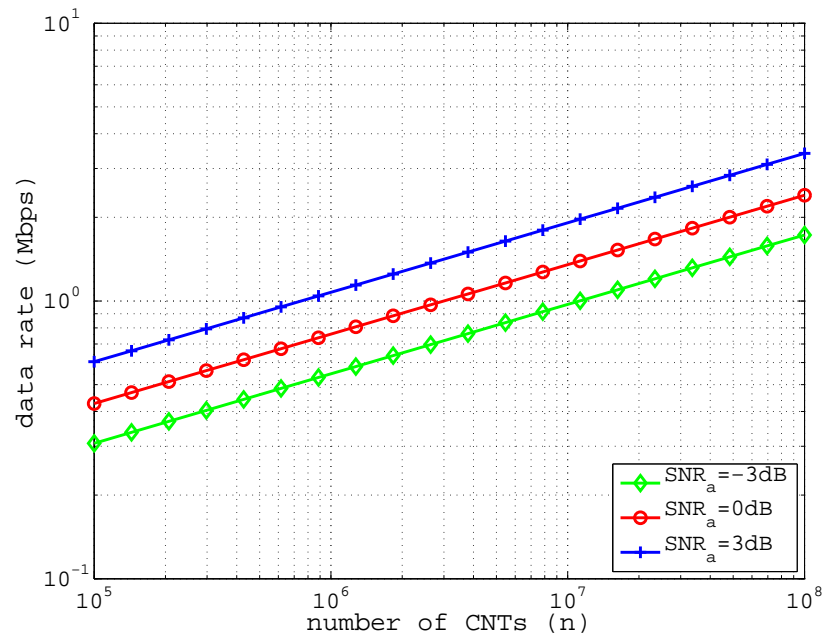

(b) Data rate vs. the number of CNTs

Fig. 5. Performance evaluation of the nanoreceiver in the high signalbandwidth regime.

the same error probability at the same SNR value is achieved with a much larger number of CNTs in the high-bandwidth regime. This is plausible since, with the increase in rate (from the low-bandwidth to high-bandwidth applications), most of the signal energy lies outside the pass-band of the CNT and gets filtered out. This loss in signal energy is made up for by the increase in the number of CNTs.

In Fig. 5(b), we analyze the achievable data rate as a function of the number, $n$, of CNTs at a fixed bit error rate (BER). We illustrate the data rate as a function of the number of CNTs in Fig. 5(b) for a BER $=10^{-5}$ for various values of $S N R_{a}$. Note that, in this regime, the achieved rate grows as $n^{1 / 4}$ at a fixed BER. At reasonably low values of $S N R_{a}$, rates of order multiple Mbps are achievable at a low BER. On the other hand, to increase the data rate by a factor of 2 , we need roughly an order of magnitude increase in the number of CNTs. Thus, for a significant increase in the rate beyond the few Mbps range, we need to either have a larger signal to noise ratio or employ error control coding with more sophisticated modution techniques than the simple OOK. Indeed, from Fig. 5(b), one can observe that a $3 \mathrm{~dB}$ coding gain gives us roughly a reduction factor of 5 in the number of CNTs at the data rate of $1 \mathrm{Mbps}$.

\section{COnClusions}

We proposed a high-rate multi-CNT nanoreceiver and analyzed its performance. The proposed system is analyzed for two different application scenarios differentiated through the bandwidth of the received signal. Low signal-bandwidth scenarios are envisioned for applications requiring lower data rates, but a robust operation for very long operation durations. These systems are observed to undergo a phase-transition with increasing number of CNTs, where error rates drop almost to zero beyond scenario-specific thresholds. We also observe that it is possible to achieve very low error rates over months of operation time with only a few thousand CNTs. For the high signal-bandwidth scenarios, we envision the development of miniaturized receivers achieving data rates comparable to those achievable by traditional RF receivers. We show that high data rates and low error rates are achievable only through large number of CNTs, which are still plausible since millions of CNTs can be planted in areas as small as fractions of a millimeter square. The data rate grows approximately proportional to the fourth root of the number of CNTs for a given bit error rate. Our results show that it is possible to achieve high reception rates at very small scales, paving the road towards a multitude of applications benefiting from the resulting miniaturization.

\section{REFERENCES}

[1] I. Akyildiz and C. B. F. Brunetti, "Nanonetworks: A new communication paradigm," Computer Networks, vol. 52, pp. 2260-2279, 2008.

[2] E. Drexler, Nanosystems: Molecular Machinery, Manufacturing, and Computation. John Wiley and Sons Inc., 1992.

[3] R. Freitas, "Nanotechnology, nanomedicine and nanosurgery," International Journal of Surgery, vol. 3, p. 243246, November 2005.

[4] — "Pharmacytes: An ideal vehicle for targeted drug delivery," Journal of Nanoscience and Nanotechnology, vol. 6, p. 27692775, September 2006.

[5] J. Aylott, "Optical nanosensors an enabling technology for intracelular measurements," Analyst, pp. 309-312, 2003.

[6] J. M. Jornet and I. Akyildiz, "Channel Capacity of Electromagnetic Nanonetworks in the Terahertz Band," Proceedings of IEEE ICC 2010, May 2010.

[7] M. Pierobon and I. Akyildiz, "A Physical End-to-End Model for Molecular Communication in Nanonetworks," IEEE JSAC, vol. 28, pp. 602-611, May 2010.

[8] M. Gregori and I. Akyildiz, "A New NanoNetwork Architecture using Flagellated Bacteria and Catalytic Nanomotors," IEEE JSAC, vol. 28, pp. 612-619, May 2010

[9] B. Behkam and M. Sitti, "Bacterial flagella-based propulsion and on/off motion control of microscale objects," Applied Physics Letters, vol. 90, January 2007.

[10] K. Jensen, J. Weldon, H. Garcia, and A. Zettl, "Nanotube Radio," Nano Letters, vol. 7, no. 11, pp. 3508-3511, November 2007.

[11] J. Weldon, K. Jensen, and A. Zettl, "Nanomechanical Radio Transmitter," Physica Status Solidi B, vol. 245, no. 10, pp. 2323-2325, October 2008.

[12] D. Dragoman and M. Dragoman, "Tunneling Nanotube Radio," Journal of Applied Physics, vol. 104, October 2008.

[13] C. E. Koksal and E. Ekici, "A Nanoradio Architecture for Interacting Nanonetworking Tasks," Elsevier Nano Communication Networks Journal, vol. 1, pp. 63-75, March 2010. 
[14] C. E. Koksal, E. Ekici, and S. Rajan, "Design and Analysis of Systems Based on RF Receivers with Multiple Carbon Nanotube Antennas," Elsevier Nano Communication Networks Journal, vol. 1, no. 3, pp. 160172 , September 2010

[15] B. Atakan and O. B. Akan, "Carbon Nanotube-Based Nanoscale Ad Hoc Networks," IEEE Communications Magazine, pp. 129-135, June 2010. 